\title{
The Challenge of Orthodonic Treatment Against Sickle Anemia: Fear or Lack of Information?
}

\author{
Luíza Trindade Vilela*, Bruna Caroline Tomé Barreto, Ana Maria Bolognese and Margareth \\ Maria Gomes de Souza
}

Department of Pedodontics and Orthodontics, Universidade Federal do Rio de Janeiro, Brazil

\section{MINI REVIEW}

Sickle cell anemia is a hematologic disease with hereditary etiology. Considered a public health issue, it has a high frequency in the population, being the most prevalent genetic disease in Brazil, especially in feoderms. The growing miscegenation in the country justifies the increase of the disease. Sickle cell anemia is a hereditary type of chronic hemolytic anemia caused by the genetic mutation of the hemoglobin molecule, where the beta globin gene originates, instead of hemoglobin A ( $\mathrm{HbA})$, an altered hemoglobin: Hemoglobin $\mathrm{S}(\mathrm{HbS})[1,2]$. The disease affects various body tissues, including the mouth.

In this situation, the orthodontic treatment of patients with sickle cell anemia becomes a major challenge, which many professionals refuse to face either for fear or for lack of information. Thus, the clarification of the aspects related to this condition is notorious.

It is of utmost importance that the orthodontist understands the condition and conducts treatment in the best way possible. Furthermore, the professional must explore family history in order to uncover the degree of systemic impairment [3].

Oral manifestations are characterized by mucosal pallor, hypercementosis, enamel and dentin mineralization disorders, eruptive delay, bone changes, malocclusions, propensity for infection, among others. Patients with sickle cell anemia present non-pathognomonic oral manifestations but may help the professional to identify the disease [4].

Radiographic changes in the bone trabeculated may be suggestive of hemoglobinopathies, enabling the professional to contribute to the early diagnosis of the disease [5]. According to Sanger [6], radiographic changes in the jaws such as the number of reduced, coarse and clearly defined bone trabeculae are indicative of sickle cell anemia. The great majority of sickle cell patients present as etiology of malocclusions disturbances and alterations of functions attributed to breathing and chewing. As a result of these damages, compensatory hyperplasia of the medullary spaces may occur, causing maxillary expansion, characterized by radiographic examinations by enlargement of the trabecular and medullary spaces. Corroborating the need for orthopedic-facial treatment of patients in these conditions.

Mucosal pallor is due to the low number of erythrocytes, as their precursors are irregularly synthesized, developing larger size (megaloblasts) and suffering apoptosis which results not turning into erythrocytes $[7,8]$.

In the spleen, there are erythrocytes with altered shapes, which are identified and destroyed, this annihilation phenomenon occurs at a faster rate than the production of these cells. Altered shape erythrocytes interfere with blood flow, since they are inflexible and obstruct microcirculation, leading to anoxia. Thus, it is recommended the use of light and continuous forces, with wider intervals between activations, as conduct adopted by the professional in order to prevent hypoxia and future tissue necrosis10. Moreover, it is essential to always respect the individuality of the patient5, providing light and continuous forces during orthodontic treatment.

In addition, the disease may cause sterile pulp necrosis due to probable pulp thrombosis of the blood vessels without a history of trauma, extensive caries or dental restorations, and may lead to bone thinning that is likely to increase over time [9], once again corroborates the indication of slower orthodontic treatment.
Quick Response Code:

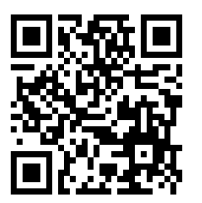

Address for correspondence: Luíza Trindade Vilela, Department of Pedodontics and Orthodontics, Universidade Federal do Rio de Janeiro, Brazil

Received: November 20, 2019 Published: December 02, 2019

How to cite this article: Luíza TV, Bruna CTB, Ana Maria B, Margareth MGdeS. The Challenge of Orthodonic Treatment Against Sickle Anemia: Fear or Lack of Information?. 2019 - 1(3) OAJBS.ID.000122. DOI: 10.38125/OAJBS.000122 
Sickle cell hemoglobinopathies are probably the most common cause of avascular bone necrosis. Chronic hemolytic anemia often causes bone marrow hyperplasia and red blood cells, leading to bone infarct [10]. Besides, osteomyelitis affecting the facial bones should be highlighted, although it is predominant in long bones. The mandible is a favorable site because of its limited blood supply and is a favorable medium for bacterial culture after vasocclusion, followed by ischemia and necrosis. Supportive therapy, antibiotics and surgery are the means of treatment for osteomyelitis [5].

Caution during the orthodontic treatment of these patients is focused on avoiding bleeding procedures during consultations. If this situation cannot be avoided, antibiotic prophylaxis should be prescribed for procedures that may cause bleeding. In the orthodontic setting, presumably simple procedures such as selecting and adapting orthodontic bands require this prevention to be performed. In addition, it is extremely important to have strict oral hygiene control and follow-up with a hematologist. Furthermore, the chronic phase of the disease is the phase of choice for dental treatment in short-term management [2]. The pain crisis is reserved only for emergency interventions [1,4]. Preventive measures aimed at mitigating the consequences of the disease should be combined with the control of factors limiting treatment, as there is no specific treatment for this condition. The use of folic acid, good nutrition and hydration, prophylaxis, early diagnosis, treatment of infections, and precaution against adverse weather conditions are some of the necessary preventive measures [11]. Treatment with multidisciplinary interaction adds benefits to the patient, contributing to the general and oral health of the individual.

Although the literature shows scarce reports, the existing ones confirm the higher rate of caries and periodontal disease in patients with sickle cell anemia. Systemic conditions contribute to this incidence, besides providing the drug interaction that causes salivary flow suppression. In addition, they cause modifications in the defense of the host. According to Brighenti [12], the higher caries index can be attributed to isolated Candida albicans biofilms obtained in patients with sickle cell anemia, which indicated higher production of lactic acid, extracellular polysaccharides and proteins when compared to healthy individuals [12]. Furthermore, reports suggesting increased severity of periodontal disease demonstrate that when comparing pocket depth in healthy individuals and sickle cell patients, the prevalence of the disease is found in the sickle cell group.

Commitment and cooperation are essential qualities for the patient during orthodontic treatment, especially when dealing with patients with sickle cell anemia. It is very common for the patient to neglect the oral condition, putting the systemic situation first. Thus, orthodontic treatment is only indicated in cases where the patient is committed to biofilm control. It is well known in Dentistry that the absence of this control configures an increase in gingival infection, which presents bleeding exacerbated by the constant hemolysis, making the infection difficult to control in a low immunity condition, added to the neglect of self-care generated by low self-esteem. Furthermore, the orthodontic appliance makes oral hygiene difficulter.

There is neglect with oral health the same way as there is with the body in general. The patient behaves carelessly aesthetically This may be justified by a possible depressive condition, frequent in this condition. Every chronic disease is a risk factor for depression, and the disease in question would not be distinct. However, depression helps detrimentally in the prognosis of sickle cell anemia. Depressive symptoms can lower the pain threshold and the individual's ability to cope with this unpleasant experience. The outcome is an increase in the intensity and frequency of pain crises, a higher number of admissions to the emergency room and hospitalizations, and lower adherence to the therapeutic scheme, with or without suicidal ideation, in adulthood [13]. In addition to contributing significantly to increased rates of caries and periodontal disease, as they lead to carelessness with body and appearance.

In view of this, it is irrefutable the fact that orthodontists abdicate the treatment of patients with sickle cell anemia due to lack of information about the disease. Similarly, the same is true for syndromic patients. Most of the time, the treatment, either interceptive or corrective, is possible, but professionals, because they do not have the knowledge and, often, do not strive to do so, neglect therapy to those in need.

Orthodontic treatment in patients with sickle cell anemia is a reality and requires special care from a local and systemic point of view, as well as control of limiting factors, resulting in improved quality of life, psychosocial aspects and increased life expectancy.

\section{REFERENCES}

1. Franco BM, Gonçalves JCH, Dos Santos CRR (2007) Buccal manifestations of sickle cell anemia and their implications in the dentistry services. Arq Odontol 43: 92-96.

2. Pithon MM (2011) Orthodontic treatment in a patient with sickle cell anemia. Am J Orthod Dentofacial Orthop 140(5): 713-719.

3. Alves PV, Alves DK, de Souza MM, Torres SR (2006) Orthodontic treatment of patients with sickle-cell anemia. Angle Orthod 76(2): 269273.

4. Sickle cell disease: oral health: prevention and care (2014) Ministry of health, Department of Hospital and emergency care - Brasília: Ministry of Health Publishing p.1-60.

5. Oral health manual on sickle cell disease (2005) Ministry of health, health care secretariat, specialized care department - Brasília: Ministry of Health Publisher p.1-52.

6. Sanger RG, Bystrom EB (1977) Radiographic bone changes in sickcle cell anemia. J Oral Med 32(2): 32-37.

7. Vinall C, Stassen LFA (2007) Dental management of the anaemic patient. J Ir Dent Assoc 53(4): 191-195.

8. Mendes PH, Fonseca NG, Martelli DR, Bonan PR, de Almeida LK, et al. (2011) Orofacial manifestations in patients with sickle cell anemia. Quintessence Int 42(8): 701-709.

9. Andrews CH, England MC Jr, Kemp WB (1983) Sickle cell anemia: An etiological factor in pulpal necrosis. J Endod 9(6): 249-252.

10. Bolarin DM, Swerdlow P, Wallace AM, Littsey, L (1998) Type I collagen as $\mathrm{n}$ marker of bone metabolism in sickle cell hemoglobinopathies. J Natl Med Assoc 90(1): 41-45.

11. Galacteros F (2001) Physiopathological basis of sickle cell disease, management and current therapeutics. Bull Soc Pathol Exot 94(2): 7779.

12. Brighenti FL, Medeiros AC, Matos BM, Ribeiro ZE, Koga-Ito CY (2014) Evaluation of caries-associated virulence of biofilms from Candida albicans isolated from saliva of pediatric patients with sickle-cell anemia. J Appl Oral Sci 22(6): 484-489.

13. Barreto FJ, Cipolotti R (2011) Depressive symptoms in children and adolescents with sickle cell anemia. J Bras Psiquiatr 60(4): 277-283. 